\title{
Performing Repetitions To Failure in Lower-Limb Single-Joint Exercise does not Reduce Countermovement Jump Performance in Trained Male Adults
}

\author{
by \\ Bruna Costa ${ }^{1}$, Danielly M. Ferreira², Petrus Gantois ${ }^{3}$, Dalton de Lima-Júnior ${ }^{3}$, \\ Witalo Kassiano ${ }^{1}$, Edilson S. Cyrino ${ }^{1}$, Leonardo S. Fortes ${ }^{3}$
}

\begin{abstract}
Performing repetitions to failure (RF) is a strategy that might acutely reduce neuromuscular performance, as well as increase the rating of perceived exertion (RPE) and the internal training load (ITL) during and after a resistance training (RT) session. Thus, this study aimed to analyze the acute effects of RF or repetitions not to failure (RNF) on countermovement jump (CMJ) performance and the ITL in trained male adults. Eleven men performed two experimental protocols in randomized order (RF vs. RNF). Under the RF condition, participants performed three sets of the leg extension exercise using 100\% of the 10RM load and rest intervals of 180-s between sets. Under the RNF condition, participants were submitted to six sets of five repetitions with the same intensity and an 80-s rest interval between sets in the same exercise. The CMJ test was analyzed before and following (15-s and 30-min, respectively) each experimental session. The ITL was evaluated by multiplying the RPE and the total session time, 30-min after the protocol. No main effect or interaction time vs. condition was found for CMJ performance $(p>0.05)$. In contrast, the ITL showed higher values under the RF condition $(p=0.003)$. Therefore, even though RF-induced a greater ITL, our results suggest that adopting this strategy in one single-joint exercise for the lower limbs does not seem sufficient to reduce CMJ height.
\end{abstract}

Key words: fatigue, resistance training, perceived exertion, psychophysiological variables, acute effects.

\section{Introduction}

Manipulating different strategies within a resistance training (RT) program is one of the key points for optimized neuromuscular adaptations (Bloomquist et al., 2013; Ikezoe et al., 2020). Although some studies (Ikezoe et al., 2020; Izquierdo et al., 2006) have shown that performing repetitions to failure (RF) does not provide additional strength, power, and hypertrophy gains compared to repetitions not to failure (RNF) performed with the same volume (number of repetitions), this strategy has been widely used for such purposes (Saric et al., 2018). However, there is still a need to improve knowledge on the acute effects of this strategy since RF might lead to increased metabolic stress markers that are directly related to the acute reduction of neuromuscular performance (Gorostiaga et al., 2012, 2014).

In this regard, Gorostiaga et al. (2012) analyzed the acute effects of RF vs. RNF in leg press exercise on neuromuscular performance and muscle metabolite markers. The results indicated that the RF condition led to a significant decrease in power output and greater increases in the muscle lactate, blood lactate, and blood ammonia concentration levels. On the other hand, the RNF condition did not change the mechanical, metabolic, and biochemical response following an RT session. Nevertheless, the neuromuscular

1 - Metabolism, Nutrition, and Exercise Laboratory. Londrina State University. Londrina, PR, Brazil.

2 - Federal University of Pernambuco, Department of Physical Education. Recife, PE, Brazil.

3 - Federal University of Paraíba, Department of Physical Education. João Pessoa, PB, Brazil. 
function may vary depending on the type of exercise performed (single- or multiple-joint). It seems that multi-joint exercises induce a higher rating of perceived exertion (RPE) when the same number of repetitions is performed, even with a greater inter-set rest interval for multi-joint exercise compared to single-joint exercise (Senna et al., 2012).

Moreover, it seems the dynamic recovery process following single- and multi-joint exercises differ according to the neuromuscular performance as post-24 $\mathrm{h}$ resistance exercise, including only single-joint exercises, provides a faster recovery process than a session adopting only multi-joint exercises (Korak et al., 2015). Since the leg extension exercise is typically prescribed in the RT routine (Ema et al., 2016), it is important to investigate the effects of the dynamic recovery process on neuromuscular performance following this single-joint exercise considering different RT strategies.

In this regard, countermovement jump (CMJ) height is a feasible and reliable test that has been used to monitor lower limb neuromuscular performance (Hiscock et al., 2018; SanchezMedina and Gonzalez-Badillo, 2011). SanchezMedina and Gonzalez-Badillo (2011) found that CMJ height loss was strongly correlated $(r=0.86$ to 0.97 ) with lactate and ammonia concentrations and velocity loss during several resistance exercise configurations. Additionally, the magnitude of $\mathrm{CMJ}$ height loss increased as the performed repetitions approached concentric muscle failure. Therefore, monitoring $\mathrm{CMJ}$ height may explain the neuromuscular status following resistance exercise to or not to failure. Moreover, it has been found that CMJ height declines as the perception of exertion during resistance exercise increases (Hiscock et al., 2018), which may occur to a greater extent following resistance exercise to failure.

Among the methods proposed to evaluate the physiological stress imposed by resistance exercise, we can highlight the internal training load (ITL) quantification. Biochemical (lactate, cortisol, creatine kinase) and autonomic responses (heart rate variability and heart rate recovery) are common strategies to analyze the ITL. However, these evaluations require specific instruments that may limit their use for strength and conditioning professionals and practitioners. Accordingly, the
session-RPE (sRPE) might be a reliable alternative method to measure the ITL with greater ecological validity and feasibility. Moreover, sRPE is considered a sensitive tool to monitor neuromuscular fatigue during RT (Hiscock et al., 2018).

Regarding the RPE evaluated during and after an RT session, studies have shown higher values when RF is adopted when compared to an RNF with equalized volume. A possible explanation for this phenomenon is that a higher RPE presents a relationship with increased adenosine and reduced dopamine in the frontal cortex (Marcora, 2009). This may be associated with higher psychophysiological stress and fatigue caused by RF (Gonzalez-Badillo et al., 2016).

Thus, the aims of the present study were: a) to analyze the acute effects of performing RF vs. RNF on neuromuscular performance of lower limbs evaluated in a different motor task using single-joint exercise and; $b$ ) to compare the effects of performing RF vs. RNF on the ITL in trained male adults. Based on previous studies (GonzalezBadillo et al., 2016), we hypothesized that the RF condition would provide a greater reduction in neuromuscular performance and higher ITL values.

\section{Methods}

\section{Participants}

The sample size analysis was conducted using $G^{*}$ Power 3.1 software. The criteria adopted were: power $(1-\beta)=0.80, \alpha=0.05$ and effect size $(E S=0.50)$. The result of the analysis showed that 10 subjects were sufficient to conduct the study. Thus, 11 men (age $=23.8 \pm 2.3$ years; body mass $=$ $78.3 \pm 6.9 \mathrm{~kg}$; height $=1.73 \pm 0.05 \mathrm{~m} ; \mathrm{BMI}=25.9 \pm$ $1.8 \mathrm{~kg} \cdot \mathrm{m}^{2} ; 10 \mathrm{RM}=98.4 \pm 13.4 \mathrm{~kg}$ ) were recruited using the non-probabilistic sampling method. Participants were selected according to the following inclusion criteria: (1) to be physically active with one to five years of consistent RT experience, particularly with the leg extension exercise; (2) male; (3) to be free from a muscular or joint injury; and (4) had not taken any ergogenic substance for strength and/or muscle mass gains over the last six months. Participants were instructed to maintain their daily routines, eating habits, and abstain from any exercise program during the study. After receiving 
information about the procedures, participants signed the informed consent form. The study was approved by the institutional Ethics and Research Committee and followed the Declaration of Helsinki's ethical principles.

\section{Measures}

Training load

The 10-repetition maximum (10RM) test for the leg extension exercise was performed following standard procedures (Brown and Weir, 2001) in two distinct sessions with a recovery time of $48 \mathrm{~h}$ to assess the reproducibility of the measure. Two attempts were made with recovery intervals of $10 \mathrm{~min}$ rest between efforts. A specific warm-up (two sets of 15-20 repetitions with 50\% of the predicted 10RM and 2 min rest intervals between sets) was performed before the 10RM test. The intraclass coefficient (ICC) and the coefficient of variation (CV) for the 10RM were considered satisfactory (ICC $=0.97$, CI 95\% $=0.92$ $0.99 ; \mathrm{CV}=2 \%$ ).

Experimental protocols

Two experimental conditions were performed (RF vs. RNF) in the present study. Under the RF condition, participants completed three sets using $100 \%$ of the 10RM load and $3 \mathrm{~min}$ rest intervals between sets. Under the RNF condition, participants performed six sets of five repetitions with the 10RM load and the 80 -s rest interval between sets. Both RT sessions included the leg extension exercise performed on a conventional weight-stack machine (Matrix ${ }^{\circledR}$, Brazil). Participants were instructed to exhale during the concentric phase and inhale in the eccentric phase while maintaining a constant velocity of movement at a ratio of approximately 1:2 (concentric and eccentric muscle actions, respectively).

\section{CMJ performance}

The CMJ was performed using a contact platform (Hidrofit ${ }^{\circledR}$, Belo Horizonte, MG, Brazil) at pre, post- $15 \mathrm{~s}$, and post-30 min experimental conditions. Participants were instructed to perform a squat with $\sim 90^{\circ}$ knee flexion with their hands on their hips. Then, the participant performed a concentric movement jump with the highest possible height and maintained the leg extension during the flight phase and landing at the same takeoff point. The jump height was calculated from the flight time according to the following equation: $9.81 \times$ flight $t^{2} \mathrm{e}^{2} / 8$. Three attempts were performed with a 30-s rest interval between each jump (Nakamura et al., 2016), and the best performance was recorded for further analysis. The ICC and the CV for the CMJ were considered satisfactory (ICC $=0.93$, CI $95 \%=0.74$ $0.98 ; \mathrm{CV}=5 \%$ ).

Perceptual measures

At the beginning of the experimental session, participants were asked about their perceived recovery status through the TQR scale proposed by Kenttä and Hassmén (1998) and validated to the Brazilian context by Osiecki et al. (2015). TQR is a scale with an anchorage that ranges from zero (not recovered) to 10 (fully recovered). Higher values mean increased recovery perception. Furthermore, participants were asked about their SRPE 30-min after each experimental session. All participants answered the following question: "How was your training session?". The response was given by means of the CR-10 indicators. The sRPE was then multiplied with the total session time in minutes to quantify the ITL (Foster et al., 2001).

\section{Design and Procedures}

This was an experimental, randomized, cross-over study of acute effects with trained male adults with RT experience. The investigation consisted of two experimental conditions (RF and $\mathrm{RNF}$ ) in the same resistance exercise (leg extension) separated by a week washout period. Simple randomization was carried out for the two experimental conditions (www.randomizer.org.site). Participants visited the laboratory on five occasions. At the first visit, all volunteers were informed about the study procedures and then signed the consent form. Next, their body mass and height were measured, and the body mass index was calculated. Finally, all participants performed the 10-repetition maximum (10RM) test to adjust the training load to be used under both experimental conditions. The 10RM test was replicated in the second visit to analyze the reproducibility. In addition, participants were familiarized with the RPE scale (CR-10 Borg) and the CMJ test. Participants then performed both experimental conditions (RF and RNF) on the fourth and fifth visits. Perceived quality of recovery was accessed by the Total Quality of Recovery (TQR) scale at the beginning of each session to avoid possible residual fatigue from the previous session. The CMJ test was 
performed before and after (15-s and 30-min) each experimental session (RF vs. RNF). The study procedures are presented in Figure 1. Sessions were conducted in the afternoon ( 2 p.m. to 5 p.m.) at the same time of day for each participant to avoid possible effects of circadian cycles on performance (Chtourou et al., 2012).

\section{Statistical analysis}

The data distribution was analyzed by the Shapiro-Wilk test, standardized skewness, and kurtoses $(+1.96$ to -1.96$)$. The paired Student's ttest was used to analyze differences in the sRPE and ITL. The Wilcoxon test was used to analyze the difference in the TQR scale. The Levene test was used to verify the homogeneity of the variances. Two-way analysis of variance (ANOVA) of repeated measures was used to analyze the interaction between conditions (RF vs. RNF) at different times (pre vs. post vs. 30-min). The effect size (ES) of the model (Partial eta squared $\left[\eta p^{2}\right]$ ) was adopted. ES for standardized mean differences was used. The ES was calculated as the RF condition mean minus the RNF condition mean divided by the pooled standard deviation (Cohen, 1992). Moreover, the relative changes were calculated using the percentage delta of the individuals' values: [(post - pre) / pre * 100]. The data were analyzed using the Statistical Package for the Social Sciences (SPSS) version 23.0 software $\left(\mathrm{IBM}^{\circledR} \quad\right.$ Corporation,
Armonk, NY, USA), and the significance level was set at $5 \%$.

\section{Results}

The perceived recovery status was similar at the beginning of both experimental conditions $(p=0.22)$ (Figure 2). CMJ performance and perceptual responses to the two experimental conditions (RF and RNF) are presented in Table 1. No significant interaction effect (condition vs. time) was observed $\left(F_{(2,18)}=2.82 ; p=0.08, \eta p^{2}=\right.$ $0.23)$. Moreover, no main effect of time $\left(F_{(2,18)}=\right.$ $\left.0.830, p=0.45, \eta p^{2}=0.08\right)$ or condition $\left(F_{(1,9)}=\right.$ 1.286, $p=0.28, \eta p^{2}=0.12$ ) was found (Figure 3). Specifically, the RF condition decreased performance by $-3.6 \%$ (CI $95 \%-9.0$ to 1.8 ; ES = $0.23)$, while the RNF condition increased it by $3.4 \%$ (CI 95\% -0.42 to 7.22 ; $\mathrm{ES}=0.16$ ) immediately after the RF condition (i.e., 15-s). In contrast, higher ITL values were identified under the RF condition $\left(t_{(10)}=-3.9\right.$; mean difference $=-122.3$ A.U; CI $95 \%-175.2$ to $-49.4, p=0.003$, ES $=0.97$ ), although the total session time was similar in both conditions $(p>0.05)$.

Individual data according to the two experimental conditions for the CMJ and ITL are illustrated in Figures 3 and 4, respectively.

Table 1

Neuromuscular and perceptual responses in trained adult men to the experimental conditions $(n=11)$. Data are presented as mean [SD]

\begin{tabular}{lcccccc}
\hline & \multicolumn{5}{c}{ Condition } \\
\cline { 2 - 7 } & \multicolumn{7}{c}{ RF } & Post & $30-$ min & Pre & Post & 30 -min \\
\cline { 2 - 7 } CMJ (cm) & $35.2[7.0]$ & $33.7[5.5]$ & $34.9[6.8]$ & $35.2[6.6]$ & $36.3[6.4]$ & $34.5[6.1]$ \\
sRPE (AU) & N/A & N/A & $7.0[1.3]^{*}$ & N/A & N/A & $5.5[1.8]$ \\
$\begin{array}{l}\text { Total session } \\
\text { time (min) }\end{array}$ & N/A & 75.2 & N/A & N/A & 76.2 & N/A \\
ITL (AU) & N/A & N/A & 533.7 & & & 421.3 \\
\hline RF & & & {$[98.5]^{*}$} & N/A & N/A & {$[130.1]$} \\
\hline
\end{tabular}

Note. $\overline{R F=\text { repetitions to failure; } R N F=\text { repetitions not to failure; } C M J=\text { countermovement jump; }}$ $S R P E=$ session rating of perceived exertion; ITL = internal training load; $A U=$ arbitrary units; $N / A=$ not applicable; ${ }^{*} p<0.05$ vs. RNF. No main effect of time, condition or interaction was revealed $(p>0.05)$. 


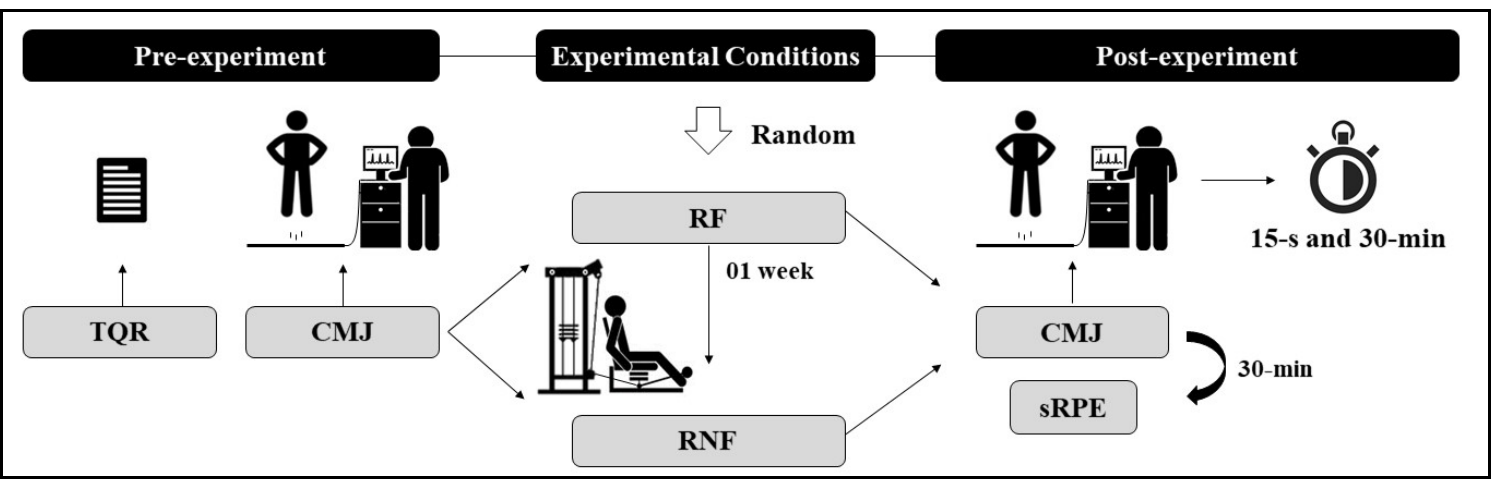

Figure 1

Study procedures. Note: $T Q R=$ total quality recovery; $C M J=$ countermovement jump; $R F=$ repetitions to failure; $R N F=$ repetitions not to failure; $s R P E=$ session rating of perceived exertion.

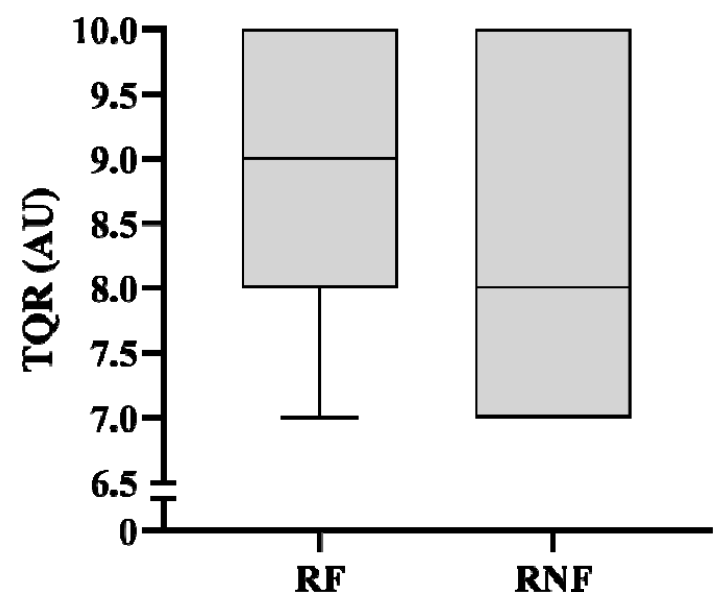

Figure 2

Box-Plot represents the recovery status of participants in median [25-75]. Note: $T Q R=$ total questionnaire recovery; $A U=$ arbitrary units; $R F=$ repetitions to failure; $R N F=$ repetitions not to failure.

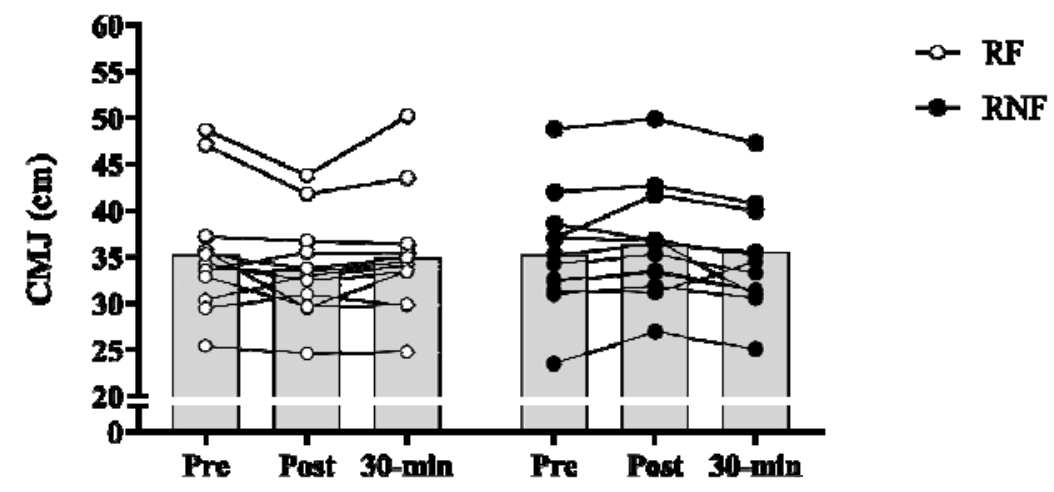

Figure 3

CMJ performance at pre, post, and 30-min. Data are presented in individual (lines) and mean (bars) values. $C M J=$ countermovement jump; $R F=$ repetitions to failure; $R N F=$ repetitions not to failure. 


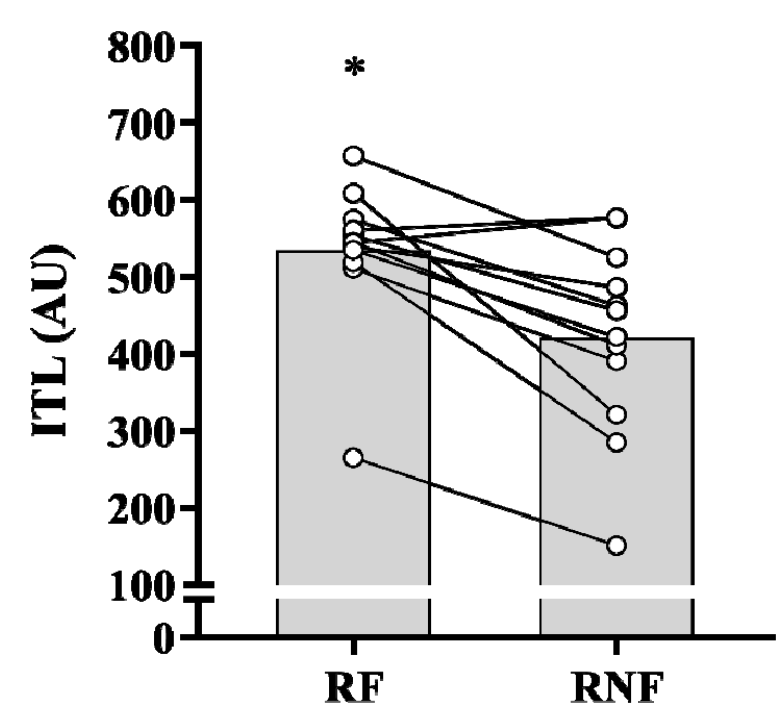

Figure 4

Individual and group data for internal training load (ITL) in the two experimental conditions are presented. Lines represent the individual values and bars represent the mean. $R F=$ repetitions to failure; $R N F=$ repetitions not to failure; $A U=$ arbitrary units. ${ }^{*} p<0.05 \mathrm{vs} . R N F$.

\section{Discussion}

This study aimed to analyze the acute effects of different RT strategies (RF and RNF) on CMJ performance and the ITL in trained male adults. The main finding of this study was that the training strategy based on repetitions to failure in the leg extension exercise did not reduce CMJ height, although higher ITL values were found following the RF condition. Therefore, our hypothesis that the RF condition would provide a greater reduction in vertical jump performance and higher ITL values than RNF was partially confirmed.

Regarding neuromuscular performance, studies have shown a reduction in acute performance after an RT session with RF (Gathercole et al., 2015; Gorostiaga et al., 2012). Indeed, it has been consistently reported that multi-joint exercise (e.g., back squat, leg press, and bench press) performed to RF induces greater metabolic, biochemical, and perceptual responses (Gorostiaga et al., 2012; Moran-Navarro et al., 2017; Pareja-Blanco et al., 2017). Despite this, our study revealed a similar CMJ performance following both experimental conditions. These results may be related to the different dynamic recovery process across multi-joint and singlejoint exercises (Korak et al., 2015), suggesting that one single-joint exercise may not acutely affect or slightly affect the ballistic exercise performance. Although the reduction in CMJ height was not statistically significant when the protocol of performing repetitions to failure was adopted, it is important to note that RF showed a decrease of $-3.6 \%$. In comparison, the RNF condition showed a $3.4 \%$ increase in test performance. Additionally, one resistance exercise per session had limited ecology validity. Therefore, future studies are warranted to include other exercises to understand the effect on neuromuscular status in a more practical setting. 
Additionally, it is important to note the rest intervals between sets and the number of exercises within the RT session can influence the acute performance responses and dictate the recovery process (Grgic et al., 2018). Findings have shown that shorter rest intervals negatively affect acute neuromuscular performance when compared to longer intervals (Ratamess et al., 2012). However, the first exercises of an RT session seem to be less affected during an inter-set performance. For example, Senna et al. (2009) compared the influence of different interval lengths on the repetitions' performance by sets. The results indicated that the number of repetitions to failure for leg extension exercise performed at the beginning of the session maintained over the three sets when individuals were submitted to longer rest intervals (i.e., five minutes). However, when the same subjects underwent shorter rest intervals (i.e., less than two minutes), the number of repetitions decreased in the second set. Thus, the longer rest interval between sets in the present study (i.e., greater than two minutes) may have dissipated the fatigue level and reduced the mechanical stress intra-set, thus enabling to maintain CMJ height, even when RF was performed.

Regarding the ITL, our results revealed that RF presented higher values when compared to the RNF condition. It is important to note that a greater ITL appears to have influenced the sRPE since the total time of the sessions was similar $(\mathrm{p}=$ 0.64). Previous findings have shown that performing RF results in an increased sRPE, even when the volume (i.e., the number of total repetitions or total session time) is equalized (Guo et al., 2017). These results are not surprising since RF leads to higher fatigue levels (Gorostiaga et al., 2012). A possible explanation for this behavior is change in exercise-induced fatigue levels, which may compromise the activity of the anterior cingulate cortex. This brain area is directly related to the RPE during exercise and consequently influences the ITL (Marcora et al., 2009). Another factor that may alter perception responses to exercise is the alteration in cerebral energy metabolism, which may also lead to an increased RPE due to the concentration of neurotransmitters antagonistic to dopamine action, influencing fatigue levels (Marcora, 2009). However, those mechanisms were not analyzed in the present study, which does not allow make such statements. Training protocols that adopt RF lead to greater sensations of pain and discomfort, which is related to the RPE (Smirmaul, 2012).

To the best of our knowledge, this is the first study that analyzed the acute effects of different strategies in RT (i.e., RF or RNF) on CMJ performance and the ITL using only one singlejoint exercise (i.e., leg extension). Our data were not supported by previous results that showed reduced neuromuscular performance when subjects underwent the RF condition. A possible explanation for these controversial results may have been due to previous studies have implemented more than one exercise for the same muscle group within the RT session (Senna et al., 2009) and/or performance of multi-joint exercises, thus increasing acute fatigue (Gonzalez-Badillo et al., 2016; Gorostiaga et al., 2014; Ratamess et al., 2012).

Although the current study presents interesting results, our data must be analyzed with caution. The experimental protocols used included only one exercise. Since individuals in the present study were previously trained, it may be that performing only one single-joint exercise was not sufficient to reduce the performance of the lower limbs substantially. In addition, responses may differ for multi-joint lower limb exercises and other body segments (e.g., upper limbs and trunk). Furthermore, due to the responses' specificity, we cannot extrapolate these findings to different populations (e.g., untrained, novice, women, older adults). Another important limitation of this study was the lack of evaluating underlying action mechanisms (muscle activation, metabolic stress markers, and electroencephalogram).

From a practical point of view, this study can help RT practitioners and trainers in the decision-making of strategies that can stimulate hypertrophic mechanisms without negatively affecting the neuromuscular component. This choice may reflect the increase in the weekly volume of training for this muscle group since the individual did not have his performance reduced. Moreover, RF should be prescribed with caution since a higher ITL was reported during the leg extension exercise. In this sense, strength and conditioning professionals and RT practitioners must keep this in mind. Future studies are 
warranted to directly compare the acute response of multi-joint and single-joint exercises to or not to failure on the neuromuscular status and include other exercises to ensure a more practical setting and increase the time course analyses (24-h and 48-h) in different resistance trained populations.

Finally, adopting strategies that need a shorter recovery period is interesting to increase the weekly frequency of training for the same muscle group and maintain mechanical efficiency during an RT session. Based on the idea that the higher weekly volume of training results in greater hypertrophic responses (Schoenfeld et al., 2017), perhaps performing RNF might be a good strategy to optimize these responses. In conclusion, performing repetitions to failure does not reduce the lower limbs' neuromuscular performance evaluated in a different motor task (CMJ) using only a single-joint exercise (leg extension). However, ITL values were higher under the RF condition when compared to the RNF in trained male adults.

\section{Acknowledgements}

We would like to express thanks to all the participants for their engagement in this study, the Coordination of Improvement of Higher Education Personnel (CAPES/Brazil) for the scholarships conceded to B.D.V.C. and P.G. (doctoral), and W.K. (master), and the National Council of Technological and Scientific Development (CNPq/Brazil) for the scientific initiation scholarship conceded to D.M.F. and post-doctoral scholarships conceded to E.S.C. and L.S.F.

\section{References}

Bloomquist K, Langberg H, Karlsen S, Madsgaard S, Boesen M, Raastad T. Effect of range of motion in heavy load squatting on muscle and tendon adaptations. Eur J Appl Physiol, 2013; 113: 2133-2142

Brown LE, Weir JP. ASEP procedures recommendation I: accurate assessment of muscular strength and power. J Exerc Physiol Online, 2001; 4: 1-21

Chtourou H, Driss T, Souissi S, Gam A, Chaouachi A, Souissi N. The effect of strength training at the same time of the day on the diurnal fluctuations of muscular anaerobic performances. J Strength Cond Res, 2012; 26: 217-225

Cohen J. A power primer. Washington: Psychol Bull, 155-159; 1992

Ema R, Sakaguchi M, Akagi R, Kawakami Y. Unique activation of the quadriceps femoris during single- and multi-joint exercises. Eur J Appl Physiol, 2016; 116: 1031-1041

Foster C, Florhaug JA, Franklin J, Gottschall L, Hrovatin LA, Parker S, Doleshal P, Dodge C. A new approach to monitoring exercise training. J Strength Cond Res, 2001; 15: 109-115

Gathercole RJ, Stellingwerff T, Sporer BC. Effect of acute fatigue and training adaptation on countermovement jump performance in elite snowboard cross athletes. J Strength Cond Res, 2015; 29: $37-46$

Gonzalez-Badillo JJ, Rodriguez-Rosell D, Sanchez-Medina L, Ribas J, Lopez-Lopez C, Mora-Custodio R, Yanez-Garcia JM, Pareja-Blanco F. Short-term recovery following resistance exercise leading or not to failure. Int J Sports Med, 2016; 37: 295-304

Gorostiaga EM, Navarro-Amezqueta I, Calbet JA, Sánchez-Medina L, Cusso R, Guerrero M, Granados C, González-Izal M, Ibañez J, Izquierdo M. Blood ammonia and lactate as markers of muscle metabolites during leg press exercise. J Strength Cond Res, 2014; 28: 2775-2785

Gorostiaga EM, Navarro-Amézqueta IN, Calbet JAL, Hellsten Y, Cusso R, Guerrero M, Granados C, González-Izal M, Ibañez J, Izquierdo M. Energy metabolism during repeated sets of leg press exercise leading to failure or not. PLoS One, 2012; 7: e40621

Grgic J, Schoenfeld BJ, Skrepnik M, Davies TB, Mikulic P. Effects of rest interval duration in resistance training on measures of muscular strength: a systematic review. Sports Med, 2018; 48: 137-151

Guo F, Sun YJ, Zhang RH. Perceived exertion during muscle fatigue as reflected in movement-related cortical potentials: an event-related potential study. Neuroreport, 2017; 28: 115-122

Hiscock DJ, Dawson B, Clarke M, Peeling P. Can changes in resistance exercise workload influence internal load, countermovement jump performance and the endocrine response? J Sports Sci, 2018; 36: 191-197

Ikezoe T, Kobayashi T, Nakamura M, Ichihashi N. Effects of low-load, higher-repetition versus high-load, lower-repetition resistance training not performed to failure on muscle strength, mass, and echo 
intensity in healthy young men: a time-course study. J Strength Cond Res, 2020; 34: 3439-3445

Izquierdo M, Ibanez J, Gonzalez-Badillo JJ, Hakkinen K, Ratamess NA, Kraemer WJ, French DN, Eslava J, Altadill A, Asiain X, Gorostiaga EM. Differential effects of strength training leading to failure versus not to failure on hormonal responses, strength, and muscle power gains. J Appl Physiol, 2006; 100: 1647-1656

Kenttä G, Hassmén P. Overtraining and recovery: a conceptual model. Sports Med, 1998; 26: 1-16

Korak JA, Green JM, O'Neal EK. Resistance training recovery: Considerations for single vs. multi-joint movements and upper vs. lower body muscles. Med Sci Sports Exerc, 2015; 8: 85-96

Marcora SM. Perception of effort during exercise is independent of afferent feedback from skeletal muscles, heart, and lungs. J Appl Physiol, 2009; 106: 2060-2062

Marcora SM, Staiano W, Manning V. Mental fatigue impairs physical performance in humans. J Appl Physiol, 2009; 106: 857-864

Moran-Navarro R, Perez CE, Mora-Rodriguez R, de la Cruz-Sanchez E, Gonzalez-Badillo JJ, Sanchez-Medina L, Pallares JG. Time course of recovery following resistance training leading or not to failure. Eur J Appl Physiol, 2017; 117: 2387-2399

Nakamura FY, Pereira LA, Cal Abad CC, Kobal R, Kitamura K, Roschel H, Rabelo F, Souza-Junior WA, Loturco I. Differences in physical performance between U-20 and senior top-level Brazilian futsal players. J Sports Med Phys Fitness, 2016; 56: 1289-1297

Osiecki R, Rubio TBG, Coelho RL, Novack LF, Conde JHS, Alves CG, Malfatti CRM. The total quality recovery $(\mathrm{TQR})$ as a proxy for determining athletes' recovery state after a professional soccer match. $J$ Exerc Physiol Online, 2015; 18: 27-32

Pareja-Blanco F, Rodriguez-Rosell D, Sanchez-Medina L, Ribas-Serna J, Lopez-Lopez C, Mora-Custodio R, Yanez-Garcia JM, Gonzalez-Badillo JJ. Acute and delayed response to resistance exercise leading or not leading to muscle failure. Clin Physiol Funct Imaging, 2017; 37: 630-639

Ratamess NA, Chiarello CM, Sacco AJ, Hoffman JR, Faigenbaum AD, Ross RE, Kang J. The effects of rest interval length on acute bench press performance: the influence of gender and muscle strength. $J$ Strength Cond Res, 2012; 26: 1817-1826

Sanchez-Medina L, Gonzalez-Badillo JJ. Velocity loss as an indicator of neuromuscular fatigue during resistance training. Med Sci Sports Exerc, 2011; 43: 1725-1734

Saric J, Lisica D, Orlic I, Grgic J, Krieger JW, Vuk S, Schoenfeld BJ. Resistance training frequencies of 3 and 6 times per week produce similar muscular adaptations in resistance-trained men.J Strength Cond Res, 2018; 33: S122-S129

Schoenfeld BJ, Ogborn D, Krieger JW. Dose-response relationship between weekly resistance training volume and increases in muscle mass: A systematic review and meta-analysis. J Sports Sci, 2017; 35: 1073-1082

Senna G, Figueiredo T, Scudese E, Baffi M, Carneiro F, Moraes E, Miranda H, Simão R. Influence of different rest interval lengths in multi-joint and single-joint exercises on repetition performance, perceived exertion, and blood lactate. J Exerc Physiol Online, 2012; 15: 96-106

Senna G, Salles BF, Prestes J, Mello RA, Simão R. Influence of two different rest interval lenghts in resistance training sessions for upper and lower body. J Sports Sci Med, 2009; 8: 197-202

Smirmaul BP. Sense of effort and other unpleasant sensations during exercise: clarifying concepts and mechanisms. Br J Sports Med, 2012; 46: 308-311

\section{Corresponding author:}

\section{Bruna Daniella de Vasconcelos Costa.}

Metabolism, Nutrition, and Exercise Laboratory. Londrina State University.

Rodovia Celso Garcia Cid km 380, Zip-code: 86057-970, Londrina, PR, Brazil.

Phone: +55 4333714772,

E-mail address: daniellavasccosta@hotmail.com 